\title{
GENETICAL AND MATERNAL DETERMINANTS OF THE ACTIVITY AND PREENING BEHAVIOUR OF DROSOPHILA MELANOGASTER REARED IN DIFFERENT ENVIRONMENTS
}

\author{
DAVID A. HAY \\ Departments of Genetics and Psychology, University of Birmingham
}

Received 23.viii.71

\section{INTRODUGTION}

THE methods of quantitative genetics are usually applied to morphological or physiological traits, but Roberts (1967) claimed that these methods could be advanced by the study of behavioural characters, which often have complex genetical and environmental bases. The present paper uses the locomotor activity and preening behaviour of Drosophila melanogaster to illustrate his point and, at the same time, to show that much information about behaviour can be obtained from a comprehensive biometrical genetical analysis.

It is well-established that the locomotor activity of $D$. melanogaster is under genetical control (e.g. Ewing, 1963; Connolly, 1966), but, with rare exceptions (Ewing, 1967), such studies have employed artificial selection, an experimental technique which has several disadvantages compared with the crossing schemes of biometrical genetics. Not only may linkage to genes affecting fitness limit the response to selection, as Siegel (1967) found when he tried to select for an optomotor response, but selection also yields less information about the genetic architecture, i.e. the magnitude of any additive variation and the magnitude and direction of dominance and nonallelic interactions. From this information we can infer how natural selection has acted on the expression of the character (Mather, 1966) and therefore whether our measurements made under artificial laboratory conditions have construct validity, that is, are we studying an aspect of behaviour important to the organism in its normal environment, rather than an artifact of our experimental situation? This question has been raised several times during the growth of psychogenetics (e.g. Murphey and Hall, 1969) and evidence that high activity may confer selective advantages in certain environments has been provided by an experiment on social behaviour (Hay, unpubl.). Testing flies in groups, rather than individually, had no effect on the performance of samples from population cages, but caused a marked decrease in the activity of inbred strains, reared at a far lower density in culture bottles where the action of natural selection is likely to be very different. The selective advantage of high activity is unknown, despite many genetical studies; Harland and Jackson (1958) attributed the advantage of white eye mutants over wild-type Drosophila in an artificial environment to the mutant's greater activity and ability to locate food but, on the other hand, Manning (1961) found a negative correlation between mating speed and activity, the most active flies reacting so strongly to all environmental stimuli that they ignored potential mates. Even less is known of the genetical control and function of preening. As a preliminary to the genetical 
analysis of this behaviour, Szebenyi (1969) distinguished 27 different forms of "cleaning" movement involving the wings, legs and body surfaces, but preening does not serve only to clean the fly, because it is subject to social influences (Hay, 1972b, and unpubl.) and may act to ward off approaching flies (Connolly, 1968).

Knowledge of the genetical architecture can also help determine the major variables influencing the behaviour of Drosophila. For example, spontaneous activity and reactivity (the response to stimulation) are controlled by independent genetical systems (Connolly, 1967), with the result that genotypic differences in activity may depend upon the type of apparatus used to test the flies (Ewing, 1967). Only small alterations of the experimental procedure are required to switch the measure of activity from one of spontaneous activity to reactivity or vice versa and it will be shown that differences in the genetical architecture provide a sensitive index of such changes, brought about by varying the test apparatus and the environment in which the flies are reared.

While biometrical genetics can thus assist studies of behaviour, it is the wide range of environmental influences on behaviour that makes behavioural traits useful in the further development of biometrical analyses. Frequently, with behavioural measures, the variances of the non-segregating generations are heterogeneous due to genotypic differences in sensitivity to the microenvironment, and it is customary to rescale the data to eliminate both this heterogeneity and any correlation between means and variances (Broadhurst and Jinks, 1961). However, in outbreeding species such as Drosophila, heterozygotes are generally less variable than homozygotes and this limited response to random environmental changes may itself be an adaptive trait (Jinks and Mather, 1955; Broadhurst and Jinks, 1966; Roberts, 1967). Using the mortality rates of different inbred lines as an index of natural selection, we shall demonstrate how a correlation between means and variances can result from selection acting on both aspects of the same trait. Although it is therefore useful to be able to analyse the variances, the difficulty arises that, without rescaling, genotype-environmental interaction can alter the second-degree statistics, in the way described by Mather and Jinks (1971) and Perkins and Jinks (1970), to such an extent that unrealistic and even negative estimates of heritability may be obtained, e.g. Broadhurst and Jinks (1961) and Newell (1970). Some alternative methods based upon first-degree statistics are available (Jinks and Jones, 1958), and the technique described in the present paper where models are fitted to the generation means is capable of evaluating most forms of gene action, irrespective of whether or not the assumptions of scaling hold.

But these models can be distorted by the influence of the maternal genotype, a problem which often arises in psychogenetics; for example, Fulker (1970) found opposing genetical and maternal effects interact to limit the response of rodent phenotypes to stress. The procedure of cross-fostering, used in mammals to control for such maternal influences, is impractical for Drosophila, where, on the other hand, the same experimental crossing programme can easily be carried out under different rearing conditions, in order to study interactions between the genetical control and the maternal genotype.

Performing the same experiment in different environments can help with one difficulty of model-fitting. Not only can maternal effects, interacting 
with the genetical influences, distort the models, but so also can statistical interaction between the genetical parameters (Perkins and Jinks, 1970). Certain parameters make equal contributions in many generations, so that their estimates are negatively correlated and inflated in opposite directions. During the fitting of models by weighted least squares, such relationships can be detected from the inverse of the information matrix (Eaves, 1969). But the practical consequences may best be gauged by comparing the models required in different conditions, where the genetical control and, hence, the observed correlations between the parameters may differ.

\section{Experimental Design}

The performance of flies from six independent crossing programmes is examined in this paper. Because strain differences in activity and preening depend on whether the flies are left in the original culture bottles until being tested 7 days after emergence or are transferred to fresh medium for this period (Hay, 1972b), the six available inbred lines were tested by $6 \times 6$ diallel crosses, including all reciprocals, carried out in both these environments. With the diallel cross, a representative sample of inbred lines can be examined (Hayman, 1954; Jinks, 1954), but, for a more detailed investigation of the two strains differing most in activity and preening, the 14 generations comprising parents $\mathrm{F}_{1} \mathrm{~s}, \mathrm{~F}_{2} \mathrm{~s}$ and first back-crosses with all possible reciprocals (see table 2) were scored in the same two environments. Unlike the diallel cross, this mating scheme permits the estimation of firstas well as second-degree genetical parameters by weighted least squares and only the former are independent of any effects on the variances due to genotype-environmental interaction. This form of analysis was first described by Cavalli (1952) and for convenience this experimental design will be referred to subsequently as the Cavalli cross.

Because it samples a greater variety of reciprocal matings with both inbred and $\mathrm{F}_{1}$ mothers, the Cavalli cross also has advantages over the diallel cross for distinguishing reciprocal differences due to sex-linkage from maternal effects. So extensive were the maternal effects in the two environments referred to above, that an additional Cavalli cross (hereafter referred to as the main Cavalli cross) was performed to determine whether these effects depended upon the culture density after eclosion or had acted upon the larvae. For this reason, the flies in this experiment were maintained in groups of the same sex at a very low density with adequate food from immediately after eclosion. The experimental size was chosen as the minimum required to reduce the standard errors sufficiently for any differences in the earlier data between the parental means and between the $F_{1}$ and the midparent to be significant; that is, to detect additive and dominance components of the means. Although the main Cavalli cross data are based, therefore, on far more flies (4480 in all) than any of the other experiments to be reported, the results of these experiments cannot be neglected, since it is unwise to draw conclusions only from flies reared in small single-sex groups. For example, the levels of activity and preening change as more flies emerge in the culture bottles and as the females reach sexual maturity (Hay, 1972a).

Unlike the other experiments described in this paper where the behaviour of the flies was recorded by time-sampling over the first 60 seconds after they had been aroused by physical stimulation, a 120 -second period was 
used in the main Cavalli cross. Activity is the most frequent response just after stimulation and the longer period of scoring was required for a separate multivariate analysis of the changing relationship between activity and preening, as the effects of the initial stimulation declined. To supplement this information, the sixth experiment was a further Cavalli cross, comparing the performance of females in two types of apparatus which differ in the intensity of the stimulation administered to the flies.

\section{Materials and methods}

For the diallel analyses, the 36 possible crosses were made between the six long-established inbred lines, Oregon, Samarkand, Florida, 6C/L, Edinburgh and Wellington, which have already served in a similar genetical analysis of male mating speed (Fulker, 1966). 6C/L and Edinburgh were chosen as the two parental strains for the Cavalli crosses, on the basis of other work (Hay, 1972a, and unpubl.), where 6G/L preened less but was more active than the other five strains, while Edinburgh displayed the opposite pattern of behaviour.

The two diallels and their corresponding Cavalli crosses were scored over a 2- or 3-day period, commencing on the seventh day after the first flies emerged from the pupal stage. All the crosses were made by mass-mating in half-pint culture bottles at $25^{\circ} \mathrm{C}$. and, in the "fresh" condition, the population of each bottle on the second day after eclosion was divided equally between two 3 in. $\times 1$ in. vials containing fresh medium; using all the flies ensured that any differences between genotypes in the number of adults in each bottle were retained among the vial populations. The testing of the diallel cross extended over 3 days, but the fresh Cavalli cross was limited to 2 days, because insufficient males survived for a third day, which was used instead for the "comparison" Cavalli cross, where only females were scored in two different types of apparatus.

In the "original" condition, the flies were left for the whole period before testing in the culture bottles where they had emerged. A high mortality was found in the diallel cross, especially among the crosses involving Oregon or Samarkand. Of the two replicates of 11 bottles comprising each array (the set of crosses involving a particular strain as at least one of the parents), seven bottles of the Oregon array and 16 of the Samarkand array lacked sufficient males for more than two successive days of testing. As the $4 \times 4$ diallel excluding these two strains was complete, it was tested on a third day and has been analysed separately from the $6 \times 6$ data, to assess the effects of the absence of these two less robust strains on the genetical control.

Because of the number of flies involved, the main Cavalli cross was divided into four blocks (two replicates of each sex), which were reared and tested independently over a period of 3 months. The following procedure was adopted in each block to control for behavioural differences between early and late emerging flies (Hay, 1972a). For all 14 generations, 10 flies of the required sex were collected within 12 hours of eclosion from each of four cultures and transferred to fresh $3 \mathrm{in} . \times 1 \mathrm{in}$. vials. The cultures were cleared of any other adults and the whole procedure repeated on the next 3 days. All these flies were tested exactly 7 days after eclosion, although, to ensure that sufficient survived for this period, it was necessary to transfer the flies to fresh vials on the fourth day. 


\section{The scoring procedure}

Many different techniques have been used to measure the activity of Drosophila, but a method had to be devised which was both simple and fast, so that data on the many individuals that are required for biometrical genetical analysis could be obtained and yet permit all the testing to be limited to the period 12.00-16.00 hours, to avoid the influence of diurnal rhythms of behaviour (Hay, 1972a). A time-sample record, where the behaviour of each fly is noted at set intervals, is a convenient way to examine activity and preening simultaneously and, after considerable practice, this method has proved to be both rapid and reliable. Some specific points about this technique are considered by Hay (in preparation).

Each fly was introduced under light $\mathrm{CO}_{2}$ sedation into a separate $10-\mathrm{cm}$. length of glass tubing, too narrow $(3 \mathrm{~mm}$. diameter) to permit flight. The tubes were randomised into groups of six or seven depending upon the experiment and each group placed on a separate tray, where partitions held the tubes steady and, more important, screened the flies from each other, lest the behaviour be influenced by those in the adjacent tubes (Ewing, 1963). After allowing a further 30 minutes for recovery, each tray in turn was placed under homogeneous illumination of 50 lux and the flies scored after being stimulated by a brisk tap on the edge of the tray. The behaviour of each fly on the tray was classified as activity, preening or inactivity at 6 -second intervals over a period of 60 seconds and over 120 seconds in the case of the main Cavalli cross. The one exception to this procedure was in the comparison Cavalli cross, where half the flies were tested instead in $18 \mathrm{~mm}$. diameter, $3.5 \mathrm{~mm}$. deep polythene dishes with a microscope coverslip as lid. The loose coverslip, originally used to permit filming of the flies (Hay, 1972b), meant that the stimulation given to the dishes had to be less intense than that used with the tubes, for fear of dislodging the lids.

Most flies were inactive at the end of the recovery period and the stimulus intensity can only be defined as that required (i) to make activity account for about 50 per cent. of all the responses among the flies tested in tubes and (ii) to ensure that the scores on both activity and preening were normally distributed. This method of determining the stimulus parameters has the advantage that it takes into account the performance of the particular group of strains being tested, rather than being determined arbitrarily. Despite the apparently crude method of applying the stimulus, its intensity did not vary significantly between the trays of tubes or dishes. Because the flies of different genotypes had been allocated at random to the trays, the " between trays" item of an appropriate analysis of variance tests for any inconsistency in the stimulation. This item was never significant in any of the experiments and we shall see that, where other sources of environmental variation were minimised, the variances between individuals within each genotype were no greater than the theoretical minima, which implies that the procedure was consistent between samples.

\section{Methods of Analysis}

Certain features of these analyses, particularly the rescaling of the diallel data and the procedure for fitting models to the generation means of the Cavalli crosses, must be described before the results can be considered.

As the scores for each fly consisted of the proportions of activity and 
preening in 10 or 20 observations, the angular transformation for small $n$ (Mosteller and Youtz, 1961) has been applied to all the data. Next, the correlation between mean and variance and the Bartlett test of homogeneity of variance were calculated for each experiment from several possible combinations of the data, for example, by considering the statistics obtained for each culture bottle separately or from the sum across all the individuals from replicate bottles within each cross. An additional transformation was then applied to the diallel data; the score of each individual was taken to the power of $(1 \cdot 0-B)$, where $B$ is the mean of all estimates of the linear regression of log mean on log standard deviation, weighted by their degrees of freedom. This transformation proved effective in eliminating any correlation between the means and variances and also much of the heterogeneity of variance, as table 1 shows. No such transformations were applied

TABLE 1

The effects of an additional transformation on the correlation between means and variances ( $\mathrm{r}$ ) and on the Bartlett $\chi^{2}$ test for homogeneity of variance among the activity and preening scores of the 72 separate groups ( 2 sexes $\times 36$ crosses) of 20 fies in the fresh condition diallel cross and the 144 groups ( 2 days $\uparrow \times 2$ sexes $\times 36$ crosses) of 6 fies in the original condition diallel cross. (For derivation of transformation, see text.)

\begin{tabular}{|c|c|c|c|c|c|c|}
\hline \multirow[b]{2}{*}{ Measure } & \multirow[b]{2}{*}{ Condition } & \multicolumn{2}{|c|}{ Before transformation } & \multirow{2}{*}{$\begin{array}{l}\text { Power of } \\
\text { transformation }\end{array}$} & \multicolumn{2}{|c|}{ After transformation } \\
\hline & & $r$ & $x^{2}$ & & $r$ & $x^{2}$ \\
\hline Activity & $\begin{array}{l}\text { Fresh } \\
\text { Original }\end{array}$ & $\begin{array}{l}-0.4989 * * \\
-0.2171 * *\end{array}$ & $\begin{array}{r}77 \cdot 62 \text { n.s. } \\
205 \cdot 88^{* * *}\end{array}$ & $\begin{array}{l}1 \cdot 8033 \\
1 \cdot 2776\end{array}$ & $\begin{array}{l}+0.0415 \text { n.s. } \\
-0.0518 \text { n.s. }\end{array}$ & $\begin{array}{l}55.89 \text { n.s. } \\
187.93^{* *}\end{array}$ \\
\hline Preenin & $\begin{array}{l}\text { Fresh } \\
\text { Original }\end{array}$ & $\begin{array}{l}+0.4856 * * \\
+0.2073 * *\end{array}$ & $\begin{array}{l}104.99 * * \\
158.88 \text { n.s. }\end{array}$ & $\begin{array}{l}0.4553 \\
0.6087\end{array}$ & $\begin{array}{l}+0.0904 \\
-0.0763 \text { n.s. }\end{array}$ & $\begin{array}{l}57.51 \text { n.s. } \\
148.95 \text { n.s. }\end{array}$ \\
\hline
\end{tabular}

*** $\mathbf{P}<0.1$ per cent.; ** $\mathbf{P}=0.1-1$ per cent.; n.s. = not significant, where the degrees of freedom are $(n-2)$ for the correlations and $(n-1)$ for the $\chi^{2} s, n$ being the number of groups.

$\dagger$ The original diallel cross data are divided into the separate days of testing because of the considerable change in performance between the two days.

to any of the Cavalli cross data, because a unique power would be required for each set of scores and would therefore prevent a direct comparison between the magnitudes of the first-degree parameters in different experiments.

Biometrical analyses were chosen which could assess the additive, dominance, non-allelic and reciprocal (sex-linked and maternal) contributions to the genetical control (for a review see Mather and Jinks, 1971). Both the North Carolina II analysis (N.C.II) and the analysis of variance of Hayman (1954) were applied to the diallel data, together with an examination of the variances and covariances $\left(V_{r}\right.$ and $\left.W_{r}\right)$ to study the direction of dominance and the form of any non-allelic interaction (Jinks, 1954). Since reciprocal differences frequently occurred, $V_{r}$ and $W_{r}$ were always calculated after averaging over reciprocal crosses and it was because of these reciprocal differences that the two analyses of variance were used. The Hayman analysis, with its " $c$ " and " $d$ " items for general reciprocal differences and specific maternal interactions respectively, provides better tests for maternal effects than the N.C.II (Wearden, 1964). The N.C.II analysis was used only to test the direction of any reciprocal differences by the ratio of the male and female array mean squares, little attention being paid to its estimate 
of non-additive effects (the male $\times$ female MS), which contains both dominance and non-allelic components, in addition to certain maternal effects, as we shall demonstrate.

In examining the Cavalli cross data, most attention is given to the firstdegree parameters fitted to the generation means by weighted least squares. However, the initial choice of these parameters depended upon the results of $\mathrm{A}, \mathrm{B}$ and $\mathrm{C}$ scaling tests for epistasis and upon comparisons between particular pairs of means, e.g. maternal effects are indicated by a difference between the reciprocal $F_{1}$ means in the female progeny. Models were fitted to the means of all 14 generations without averaging over reciprocals, to ensure that several degrees of freedom remained for a $\chi^{2}$ test of goodness of fit, and in all cases each of the 14 means was weighted by the inverse of its own variance rather than by a common weight, because the variances frequently differed significantly even within the same backcross or between reciprocal $\mathrm{F}_{1} \mathrm{~s}$ (see table 5, later). A maximum of 10 parameters were considered and their coefficients in each generation are given in table 2.

\section{TABLE 2}

Model specifying the ten parameters fitted to the generation means. (For definitions of parameters see text.)

$$
\begin{aligned}
& \text { Parameters }
\end{aligned}
$$

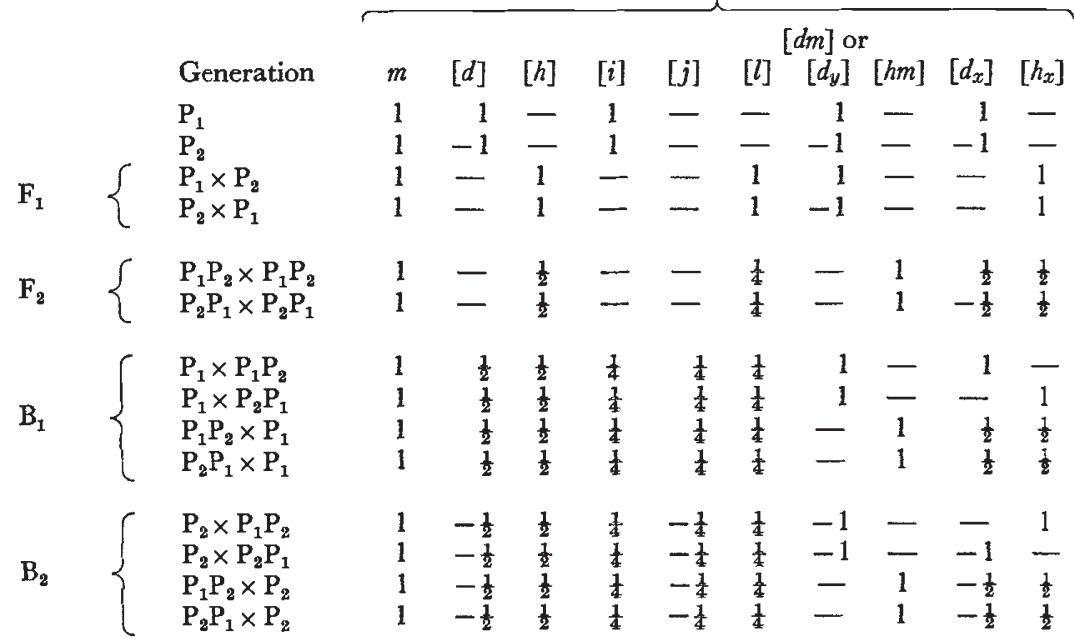

The overall mean is $m$, while $[d]$ and $[h],[d m]$ and $[h m]$ and $\left[d_{x}\right]$ and $\left[h_{x}\right]$ are the additive and dominance parameters for the autosomal, maternal and sex chromosome contributions respectively, where the notation refers to the effects across all loci, regardless of the degree of association or dispersion. Digenic non-allelic interactions are specified in the $F_{\infty}$ metric by the terms $[i],[j]$ and $[l]$, which are the homozygote-homozygote, homozygoteheterozygote and heterozygote-heterozygote interactions respectively, across all pairs of loci at which the inbred lines differ. No more than eight parameters may be fitted to the male progeny data, as firstly there can be no dominance on the sex chromosome, that is, no $\left[h_{x}\right]$ contribution to the mean, and secondly, the parameter $\left[d_{y}\right]$ for the effect of the hemizygous sex chromosome has the same coefficients as the additive maternal effect $[d m]$. A more 
detailed description of the parameters is provided by Mather and Jinks (1971).

Deciding which parameters are required to specify any set of generation means is a deceptively simple procedure. The sum of squares for the difference between the observed and expected generation means is distributed as a $\chi^{2}$, with degrees of freedom equal to the number of generation means minus the number of parameters estimated, and the fitting of models can be continued until this $\chi^{2}$ is minimised. Although the successive modelfitting procedure is fully justified by Jinks and Perkins (1969), there sometimes had to be a compromise over the parameters to be included. In general, non-significant parameters have been removed and reduced models fitted, but such parameters were retained in cases where the $\chi^{2}$ was increased significantly by their deletion or where the comparisons of generation means indicated they were relevant to the data.

This problem can best be appreciated from the results, but one contributing factor was the effect of genotype-environmental interaction on the variances of the generation means, which were used to calculate the weights for the model-fitting, as well as providing standard errors for the scaling tests and the comparisons among generation means. Models could not be fitted to the second-degree statistics since there was no obvious segregational variance, due, as we shall see, to differences between genotypes in their sensitivity to microenvironmental infuences rather than to lack of genetical control. There are several environmental levels at which such interaction could have occurred, for instance, within each culture bottle or between replicate bottles. At each such level, $j$, the variances of the parental means can be specified for a single locus as $E_{j}+G_{j D} \pm 2 \operatorname{cov} E_{j} G_{j D}$, where $E_{j}$ and $G_{j D}$ are, respectively, the additive environmental influence and the interaction of the environment with the additive effects of the gene. The variance of the $\mathrm{F}_{1}$ is $E_{j}+G_{j H}+2 \operatorname{cov} E_{j} G_{j H}$ and all other generations can similarly be specified in terms of $G_{j D}$ and $G_{j H}$ (Perkins and Jinks, 1970). However, this interaction can best be studied in the diallel crosses which do not involve segregational variation, and therefore N.C.II and Hayman analyses have been applied to the diallel variances after the scores had undergone a $\log _{10}$ transformation, in addition to the angular and the additional transformations described previously.

Such disturbances of the variances are no bar to the fitting of models to the generation means, since the standard error for each parameter is based on the entire variance-covariance matrix. The relative sensitivities of the three scaling tests for epistasis are altered, because their S.E.s are derived from fewer generations, for example $V_{A}=4 V_{\bar{B}_{1}}+V_{\bar{F}_{1}}+V_{\bar{P}_{1}}$ and $V_{\bar{B}}$ $=4 V_{\bar{B}_{2}}+V_{\bar{F}_{1}}+V_{\bar{P}_{2}}$. In the presence of interaction between the additive genetical effects and the microenvironmental influences, there will be a difference of $4\left(\operatorname{cov} E G_{D}+\operatorname{cov} G_{D} G_{H}\right)$ between the variances of the $\mathrm{A}$ and B tests.

\section{Results}

The models that best fitted the generation means of the activity and preening data are given in table 3 for all four Cavalli crosses.

\section{Activity}

A simple additive and dominance genetical model is sufficient to describe the activity of the females in the main Cavalli cross (table $3 a$ ). While there 


\section{TABLE 3}

The models required to describe the generation means for activity and preening in the four Cavalli crosses (described in text), with the $\chi^{2}$ tests of goodness of fit. The parameters are fitted to the angular-transformed data and the total number of flies upon which each model is based are given in parentheses beside the title of each experiment.

(a) Main Cavalli cross-flies reared at a uniform low density (1120)

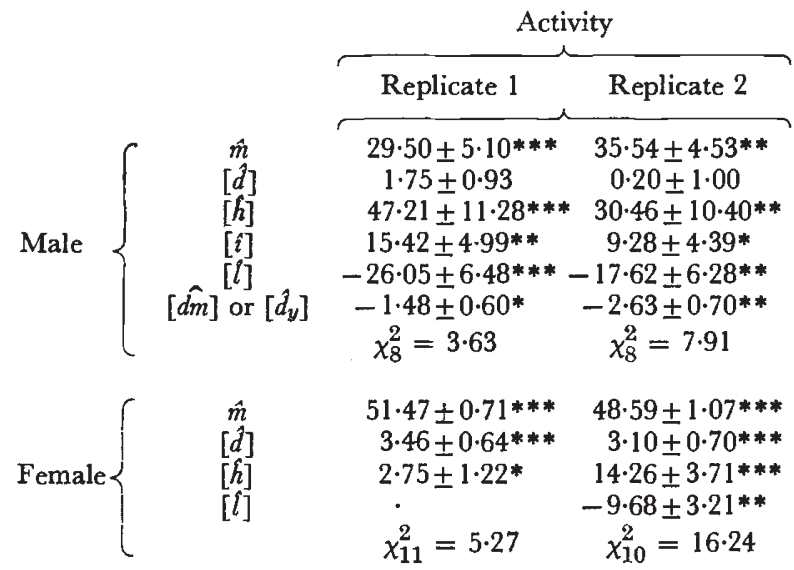

\begin{tabular}{|c|c|}
\hline \multicolumn{2}{|c|}{ Preening } \\
\hline Replicate 1 & Replicate 2 \\
\hline $29.94 \pm 0.33 * * *$ & $31 \cdot 22 \pm 0.35 * * *$ \\
\hline $3.62 \pm 0.63^{* * *}$ & $1.81 \pm 0.91 *$ \\
\hline$\cdot$ & $\cdot$ \\
\hline • & - \\
\hline - & • \\
\hline$\cdot$ & $1 \cdot 81 \pm 0 \cdot 66 * *$ \\
\hline$\chi_{12}^{2}=23 \cdot 26 *$ & $\chi_{11}^{2}=9 \cdot 06$ \\
\hline $27 \cdot 77 \pm 0 \cdot 66^{* * *}$ & $29.63 \pm 0.82 * * *$ \\
\hline $4 \cdot 65 \pm 0.58 * * *$ & $3.97 \pm 0.56 * * *$ \\
\hline$-2 \cdot 37 \pm 1 \cdot 15^{*}$ & $-6 \cdot 94 \pm 2 \cdot 90^{*}$ \\
\hline i & $5 \cdot 80 \pm 2 \cdot 64 *$ \\
\hline$\chi_{10}^{2}=9 \cdot 82$ & $\chi_{10}^{2}=9 \cdot 36$ \\
\hline
\end{tabular}

(b) Flies in fresh vials $(540) \dagger$

\begin{tabular}{|c|c|c|c|c|}
\hline & & & Pre & \\
\hline & Male & $\underbrace{\text { Female }}$ & $\underbrace{\text { Male }}$ & Female \\
\hline$\hat{m}$ & $52 \cdot 36 \pm 1 \cdot 57 * * *$ & $48 \cdot 79 \pm 2 \cdot 03 * * *$ & $23 \cdot 88 \pm 0.54 * * *$ & $23.93 \pm 0.75 * * *$ \\
\hline$[\hat{d}]$ & $4 \cdot 61 \pm 2 \cdot 42$ & $\cdot$ & $\cdot$ & $\cdot$ \\
\hline$[\hat{h}]$ & $6.92 \pm 2.50 * *$ & $8 \cdot 00 \pm 3 \cdot 17 * *$ & - & $\cdot$ \\
\hline$[j]$ & $-14.92 \pm 5 \cdot 78 * *$ & $\cdot$ & $\cdot$ & $\cdot$ \\
\hline$\hat{d m}]$ or $\left[\hat{d}_{y}\right]$ & $2 \cdot 23 \pm 1 \cdot 12 *$ & . & $2 \cdot 06 \pm 0 \cdot 69 * *$ & $2 \cdot 48 \pm 0.75 * * *$ \\
\hline$[\hat{h m}]$ & · & $6 \cdot 11 \pm 1 \cdot 59 * * *$ & $\cdot$ & $-1 \cdot 72 \pm 1.09$ \\
\hline$\left[\hat{d}_{x}\right]$ & - & $5 \cdot 51 \pm 1 \cdot 36 * * *$ & - & $\cdot$ \\
\hline$\left[\hat{h}_{x}\right]$ & - & $\cdot$ & - & ; \\
\hline & $\chi_{9}^{2}=6.99$ & $\chi_{10}^{2}=13.30$ & $\chi_{12}^{2}=23 \cdot 37^{* *}$ & $x_{11}^{2}=11.57$ \\
\hline
\end{tabular}

$\dagger$ The parameter $\left[d_{y}\right]$ is specific to males and $\left[d_{x}\right]$ and $\left[h_{x}\right]$ to females.

(c) Comparison of females in two forms of apparatus (168)

Activity

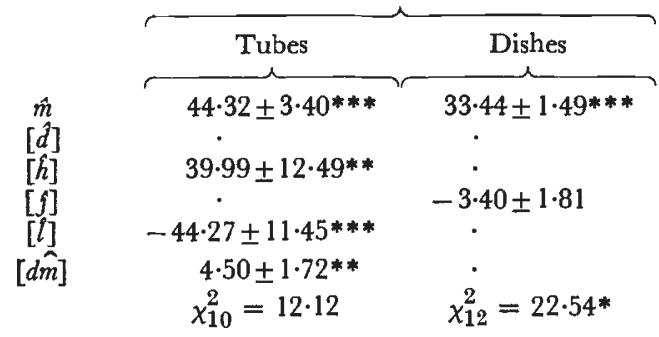

(d) Flies left in original culture bottles (756)

Activity

\begin{tabular}{|c|c|c|}
\hline & Male & Female \\
\hline $\begin{array}{c}\hat{m} \\
{[\hat{d}]} \\
{[\hat{h}]}\end{array}$ & $\begin{array}{c}50 \cdot 18 \pm 1 \cdot 42^{* * *} \\
3 \cdot 18 \pm 1 \cdot 25^{* *} \\
-3 \cdot 89 \pm 2 \cdot 44\end{array}$ & $\begin{array}{l}49 \cdot 22 \pm 0 \cdot 62 * * * \\
8 \cdot 26 \pm 1 \cdot 66 * * * \\
\quad\end{array}$ \\
\hline$[d \hat{m}]$ & $\cdot$ & $-3 \cdot 82 \pm 1.26 * *$ \\
\hline$[\hat{\mathrm{m}}]$ & $\dot{\chi}_{11}^{2}=15.95$ & $x_{11}^{2}=13.25$ \\
\hline
\end{tabular}

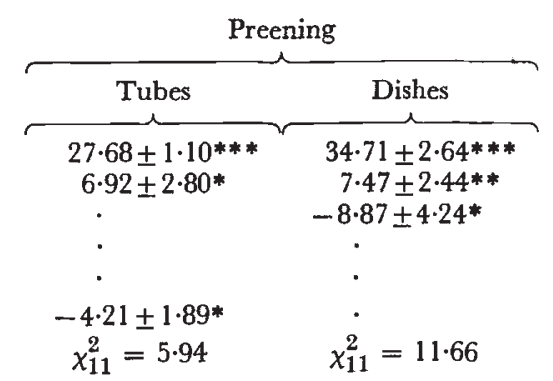


appears to be non-allelic interaction in Replicate $2,[\hat{h}]$ and $[\hat{\imath}]$ are negatively correlated because they have similar coefficients in many of the generations (table 2). Their estimates are therefore less reliable and may be inflated in opposite directions, increasing the probability of detecting spurious interaction. The sex chromosome may influence activity, since the addition of $\left[d_{x}\right]$ and $\left[h_{x}\right]$ to the model for Replicate 2 improved the goodness of fit by $\chi_{2}^{2}=5 \cdot 76$, giving $[\hat{d}]=1 \cdot 36 \pm 1 \cdot 34$ and $\left[\hat{d}_{x}\right]=1 \cdot 57 \pm 1 \cdot 13$, whose sum is close to $[d]=3 \cdot 70 \pm 0 \cdot 70$ in the simple model shown in table $3 a$.

However, no maternal effects were found in the female data, so that the parameter $[d m]$ or $\left[d_{y}\right]$ in the males confirms the presence of sex linkage and is not due to the maternal genotype. The males are far less active than the females, but a high level of activity in most generations is ensured by directional dominance plus duplicate interaction. Because $[d]=r_{d} \Sigma d,[\hat{\jmath}]$ $=r_{j} \Sigma_{j}$ and $[i]=\frac{\left(k r_{i}^{2}-1\right) \Sigma i}{k-1}$, where $k=$ number of loci and $r=$ degree of association or dispersion, then as $r \rightarrow 0$, i.e. to complete dispersion, $[d]$ and $[\hat{\jmath}] \rightarrow 0$ and $[\hat{\imath}] \rightarrow \frac{-1 \Sigma i}{k-1}$, reversing the sign. So, because $[\hat{h}]$ and $[\hat{\imath}]$ are both positive, but $[\hat{\imath}]$ is negative, there must be duplicate interaction with genes in dispersion. Although the heterosis for high activity in the male progeny may only be the result of the inflation of $[\hat{h}]$ and $[\hat{\imath}]$ by their statistical correlation-compare for example the difference in $[\hat{h}]$ between the two replicates of the female data-it is more probably the outcome of the reduction of $[d]$ through this dispersed gene distribution. Certainly additive effects must exist before there can be [i] type in teractions between the homozygous genes at different loci. Unfortunately, the distortion of the variances (see table 5, later) did not permit estimates of the second-degree statistics, $D$ and $H$, and hence of the dominance ratio $H / D$, which, unlike the potence ratio $[h] /[d]$, is independent of the degree of association or dispersion.

The fresh Cavalli cross (table $3 b$ ), where the flies were reared in culture vials at a far higher density, confirms these findings, including the influence of sex-linkage and epistasis on the activity of females. A significant positive value of [i] could be obtained in the female model, but it is confounded statistically rather than genetically with $[\hat{h m}]$, so that when both were fitted in the same model, the value of $m$ became unrealistically large. We can turn to the results of the A, B and C scaling tests in table 4 for proof that

TABLE 4

$A, B$ and $C$ scaling tests on the activity data, averaged over males and females in the fresh Cavalli cross, and their expectations for many pairs of genes

\begin{tabular}{lcll} 
s & Result & \multicolumn{1}{c}{$\mathrm{P}$} & \multicolumn{1}{c}{ Expectation } \\
$\mathrm{A}$ & $-7 \cdot 61 \pm 5 \cdot 16$ & n.s. & $\frac{1}{2}(-[i]+[j]-[l])$ \\
$\mathrm{B}$ & $+3 \cdot 57 \pm 5 \cdot 08$ & n.s. & $\frac{1}{2}(-[i]-[j]-[l])$ \\
$\mathrm{C}$ & $-19 \cdot 23 \pm 9 \cdot 57$ & $<5 \%$ & $-2[i]-[l]$
\end{tabular}

there is duplicate interaction with a dispersed gene distribution. From the expectations of these tests we see that the $\mathrm{C}$ test can differ from zero only in the presence of $[i]$ or $[l]$, not $[\hat{\jmath}]$, type interactions and so the absence of $[l]$ from the models of table $3 b$ would indicate the [i] type effect, that could not be reliably estimated in the female data. Although neither the A nor 
B tests were significant, possibly because of the effects on their S.E.s of genotypic differences in the variances (see table 5, later), they deviated from zero in the expected directions since:

(i) the $\mathrm{A}$ and $\mathrm{B}$ tests should be of opposite sign for the [ $j]$ type interaction which is found in the males (table 3 );

(ii) if there is duplicate interaction and dominance for high activity, $[j]$, and hence the A scaling test, must be negative; and

(iii) the negative value for the $\mathrm{C}$ test shows that [i] must be positive (as found in the female model $)$. But $[\hat{\jmath}]$ is negative, so that for any consistent form of gene interaction to be present, the sign of [i] must be reversed by gene dispersion, another possible explanation for the failure of the A and B tests to detect epistasis (Mather and Jinks, 1971).

The parameter [i] was confounded with $[\mathrm{hm}]$, but any environmental effect of the mother, either $[\mathrm{dm}]$ or $[\mathrm{hm}]$, is more likely than the genetical parameters to fluctuate over the days of testing. The females scored in tubes in the comparison Cavalli cross provide data for a third day of testing and here the dominance maternal effect has been replaced by an additive effect and the negative [i] shows duplicate interaction is present (table $3 c$ ). This interaction is also shown by the item $[j]$, when the females were tested in dishes, but no adequate model could be obtained for these data, although the same comparisons among the generation means-between the $F_{1}$ s and between $F_{1}$ and $F_{2}$-were significant in both conditions. These comparisons indicate the presence of $[\mathrm{dm}]$ and $[\mathrm{hm}]$ respectively (table 2) and each of the parameters in an $m,[d],[h],[d m]$ and $[h m]$ model reduced the $\chi^{2}$ significantly, despite none of them being significant against their own standard error. The $m,[j]$ model provided the best compromise but we can conclude the autosomal and maternal effects interact, as the preening data will demonstrate even more clearly.

Because it is based on six rather than just two inbred lines, the diallel cross allows us to evaluate the generality of the information from $6 \mathrm{C} / \mathrm{L}$ and Edinburgh. No additive variation was found, but potence for high activity was indicated in the Hayman analysis by a significant $b_{1}$ MS $(\mathbf{P}<1$ per cent.) -22 of the $30 \mathrm{~F}_{1} \mathrm{~s}$ in both sexes being more active than either parent -and sex-linkage by reciprocal differences confined to the males (the $c \times$ sex $\mathrm{MS}, \mathrm{P}<5$ per cent.). Because the data were averaged over reciprocals before calculating the variances and covariances, the regression of $W_{r}$ on $V_{r}$ provides a test for epistasis which is free from the problem in model fitting of confusion with maternal effects. There was no epistasis in the females, since the regression slope of $b=0.6562 \pm 0 \cdot 1672$, d.f. $=4$ does not differ significantly from $1 \cdot 0$. However, there were sex interactions of both the $b_{2}$ and $b_{3}$ items in the Hayman analysis $(\mathrm{P}<5$ per cent. and $\mathrm{P}<1$ per cent. respectively) and the type of departure from linearity shown by the males $(b=0.3624 \pm 0.2630$ and fig. 1$)$ indicates duplicate interaction, since the four intermediate arrays have negative $W_{r}$ values and cluster together (Mather, 1967). However, this distribution of the points may also be due in part to the dispersed gene distribution found previously in Edinburgh and $6 \mathrm{C} / \mathrm{L}$, as dispersion can lead to the array points showing a random scatter through the central region of the $W_{r} / V_{r}$ graph (Hill, 1964).

The genetical architecture is very different when the flies are left in the 
original bottles for at least 7 days before being tested. The analyses in table $3 d$ show that the dominance for high activity has been lost in both sexes and that the sign of $[d \hat{m}]$ in the females is reversed, compared with the comparison Cavalli cross. These changes were not restricted to $6 \mathrm{C} / \mathrm{L}$ and Edinburgh, as additive and maternal effects now influence the activity of the female progeny in the $6 \times 6$ diallel cross (the $a$ and $c$ MSs, both $\mathrm{P}<1$ per cent.) and the direction of dominance is reversed; the regression of $W_{r}+V_{r}$ on the parental means is $+14 \cdot 17 \pm 5 \cdot 28$, compared with $-121 \cdot 67 \pm 60 \cdot 24$ in the fresh diallel cross. A significant $d \mathrm{MS}(\mathrm{P}<5$ per cent. $)$ indicates that some of the reciprocal effects are restricted to particular crosses and, in the analysis of the $4 \times 4$ diallel data excluding Oregon and Samarkand, no items were significant on the three days of testing, but the $a, d$, and male parent mean squares all changed significantly $(\mathrm{P}<1$ per cent.) during this period.

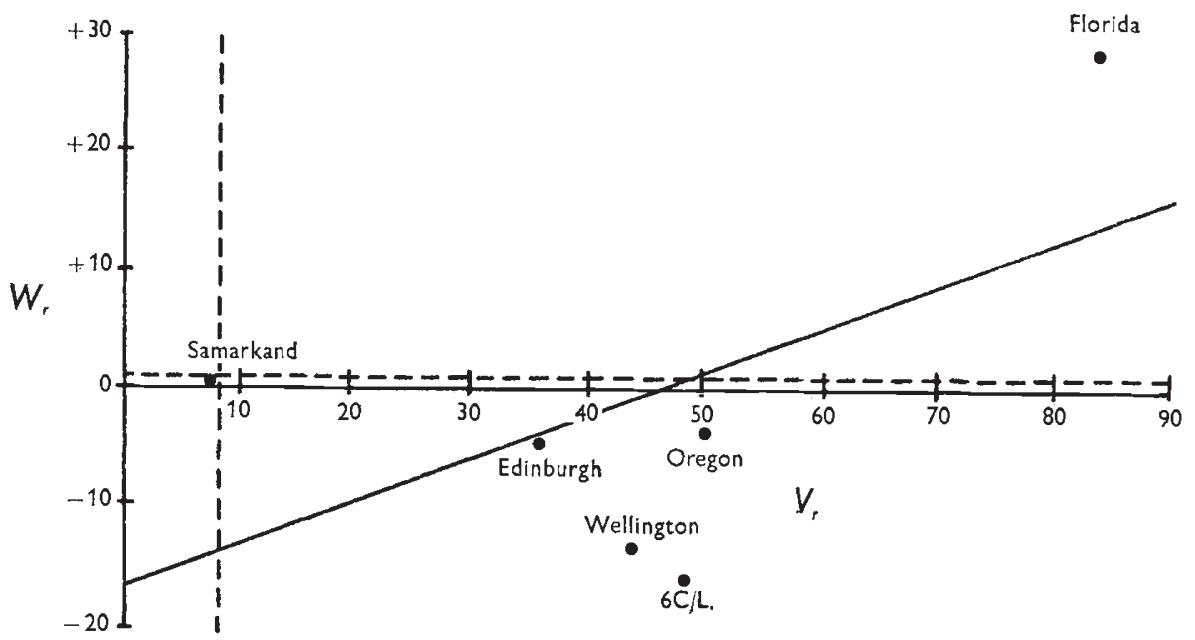

Fig. 1.-Regression of $W_{r}$ on $V_{r}$ for the activity of males in the diallel cross in fresh vials. These statistics were calculated from the data after the additional transformation (table 1) and both the original axes (- - , and the axes after appropriate $E_{2}$ corrections $(-----)$, are shown.

The N.C.II analyses of each of the three days separately showed that the father's genotype was becoming increasingly important, but, while a reciprocal difference may be ascribed to the paternal genotype statistically, it is unlikely biologically. Fulker (1970) encountered the same phenomenon in the open-field behaviour of mice and it highlights one disadvantage of the N.C.II analysis. In the presence purely of additive genetic variation with no reciprocal differences, the male and female parent mean squares should be identical; but if there is a maternal effect acting in the opposite direction to the additive difference between the lines, the variance of the female parental array will be reduced and there will appear to be a paternal effect.

In both the diallel and Cavalli cross experiments, activity rose significantly $(P<0.1$ per cent.) over the three days of testing and the diallel genotype-environmental interaction analysis of Perkins (1970) was used to find if a single inbred strain or $F_{1}$ were responsible for the increased activity. 
The two reciprocal $\mathrm{F}_{1} \mathrm{~s}$ between $6 \mathrm{C} / \mathrm{L}$ and Edinburgh gave significant values of $\beta_{h}\left(\beta_{h}=-2 \cdot 6671 \pm 0.8166, \mathrm{P}<5\right.$ per cent.), indicating that dominance was reduced as the environmental values (based only on the parental means) rose. A parallel effect was found in the Cavalli cross where the loss of the dominance for high activity was due to a rise in the activity of the $6 \mathrm{C} / \mathrm{L}$ parent from 42.23 on the first day of testing (expressed as the proportion in angles of all responses) to 57.49 and 62.14 on the second and third days respectively, while Edinburgh and the $F_{1}$ s were little affected. So, $[d]$ was increasing, but the models fitted to the data for each of the three days showed that this effect was being counteracted to some extent by a maternal effect $[d m]$ of opposite sign. A similar phenomenon in the diallel cross would, as noted earlier, reduce the female parent mean square and explain the apparent "paternal " effect.

On the other hand, such changes in activity during the course of the testing did not occur in the males. Potence for low activity was always present in the Cavalli cross (table $3 d$ ) and in both the $6 \times 6$ and $4 \times 4$ diallel cross analyses, because the means of the inbred lines but not the $F_{1}$ s had risen compared with their performance in the other conditions of rearing.

\section{Preening}

Although the time-sampling techniques has many advantages over other forms of measurement, it means that a fly which is active cannot simultaneously be recorded as preening and therefore that the scores for each individual on the two measures need not be independent. Table $3 a$ shows that the models for the preening of females in the main Cavalli cross are mirror-images of those for activity discussed earlier with additive genetic differences and dominance in Replicates 1 and 2, plus [ $l]$ type interactions in Replicate 2.

No such similarity between activity and preening is found among the males. In Replicate 2 there are both additive and maternal influences on preening, and an interaction between $[d]$ and $[d m]$ may be responsible for the failure to obtain an adequate model for Replicate 1 ; while an $m$, [d] model for Replicate 2 gave a $\chi_{12}^{2}$ of 16.67 with $[\hat{d}]=3.60 \pm 0.65$, which is identical to the estimate of $[d]$ in Replicate 1 , the replicates differed in that the addition of $[d m]$ did not reduce the $\chi^{2}$ in Replicate 1. Mather and Jinks (1971) specified three forms of interaction in the presence of maternal effects :

(i) between the maternal effect and the progeny genotype;

(ii) between the genes in the mother, responsible for the maternal effect; and

(iii) between the non-allelic genes in the progeny genotype.

These three may be distinguished by which of the A, B or C scaling tests fail, when carried out separately on each reciprocal set of crosses. If none of the three tests differ from zero, as happened in these data, the appropriate model will involve interactions between the maternal effects and the progeny genotype, with parameters such as $[d . d m]$ and $[h . d m]$, although further generations such as second back-crosses would be required to estimate their magnitude.

Most maternal effects in Drosophila reflect some aspect of the conditions of rearing rather than cytoplasmic inheritance and table $3 b$ clearly shows 
that maternal effects are a more important influence on preening in the fresh Cavalli cross, where each of the 14 generations was maintained in vials, not in uniform numbers, but at a density related to that normally found in their culture bottles. On this occasion, the inadequacy of the male model was the result of non-allelic interaction between the genes of the progeny genotype, because the A scaling test with Edinburgh as mother, that is,

$$
\mathrm{A}=(\mathrm{E} \times \mathrm{EG})+(\mathrm{E} \times \mathrm{CE})-(\mathrm{E} \times \mathrm{E})-(\mathrm{E} \times \mathrm{C}),
$$

was significant at the 1 per cent. level. The failure of this test leaves no way to determine whether interaction between the maternal and progeny genotypes, as found in the main Cavalli cross, is contributing to the $\chi^{2}$ of the model, since we cannot estimate the additional parameters from our present set of generations. However, the female data illustrate the way maternal influences can minimise the normal autosomal additive differences between the generations. In the fresh Cavalli cross model based on two days of testing, $[\hat{d}]$ is not significant, but the estimate of $[\hat{d m}]$ is equal to the sum of $[\hat{d}]$ and $[\hat{d m}]$ on the following day (the tube model of the comparison Cavalli cross in table $3 c$ ), where the $\chi^{2}$ is considerably smaller.

On the same basis, maternal and autosomal effects acting in opposite directions would explain why no additive or maternal effects were detected in the analysis of the female progeny of the diallel cross, in contrast to the males where there were both additive effects (the $a \mathrm{MS}, \mathrm{P}<1$ per cent.) and, unlike the Cavalli cross, potence for low preening (the $b_{1} \mathrm{MS}, \mathrm{P}<5$ per cent.). Although neither the $c$ nor $d$ items of the Hayman analysis were significant, interaction between the maternal and progeny genotypes may still be present, because such interaction affects the estimates of the autosomal additive and dominance components in the N.C.II, but not in the Hayman analysis of variance (Mather and Jinks, 1971). This effect would explain why the N.C.II analysis of the male data gave no indication of additive or dominance variation, only the female parent MS being significant and $3 \times$ the male parent MS, despite the fact that potence must be present since 20 of the $30 \mathrm{~F}_{1} \mathrm{~s}$ preened less than either parent and only three more than the midparent.

Preening is increased and there is dominance for low preening among the females tested in dishes in the comparison Cavalli cross where they received less initial stimulation than in tubes (table $3 c$ ). Thus the lower estimates of the mean and the absence of dominance for low preening in the fresh diallel and Cavalli cross analyses of females, compared with the main Cavalli cross, are not the result of the different rearing conditions but rather of the longer period of observation in this last experiment, during which the effects of the initial stimulus will diminish.

The inferior environment of flies reared in the original culture bottles also reduces the responsiveness of females to stimulation, since the original Cavalli cross model for the female progeny (table $3 d$ ) closely resembles that of flies reared in fresh vials, but tested in the dishes instead of the usual tubes. If dominance for low preening does result from the effects of the poor environment, then it should increase over the three days of testing as the conditions deteriorate even further, and such a change occurred both in the Cavalli cross and in the $4 \times 4$ diallel cross (the day $\times b_{1}$ MS, $\mathrm{P}<0.1$ per cent.).

Any maternal effects found in the fresh conditions which are related to the density of flies in the cultures should be accentuated by leaving the flies 
in the original bottles, where differences in numbers would, for example, influence the extent of competition for the limited food. The [hm] parameter for the contribution of an $\mathrm{F}_{1}$ mother, which had been included in the fresh Cavalli cross model because it reduced the $\chi^{2}$, now has a significant effect on the preening of females. In models fitted to the generation means of the male progeny for the three successive days of testing, the additive maternal effect $[\mathrm{dm}]$ became increasingly important and similarly, in the Hayman and N.C.II analyses of the $6 \times 6$ diallel cross, the day $\times d$ and day $\times$ male $\times$ female interactions were significant at the 1 per cent. level. It should be noted that this maternal effect, because it involves interaction with specific crosses (the $d$ item), is shown in the N.C.II analysis by a change of the male $\times$ female MS, while in the corresponding examination of female activity, the male MS of this analysis was affected, since there the additive and maternal effects were counteracting each other. Although this interaction of reciprocal differences was found in the $4 \times 4$ as well as the $6 \times 6$ diallel cross, maternal effects were generally of far greater importance in both sexes when Oregon and Samarkand were included in the analysis, since the $c$ MS was significant at $\mathrm{P}<0 \cdot 1$ per cent. in the $6 \times 6$ diallel cross, but was not significant in the $4 \times 4$ cross.

\section{The analyses of the inter-individual variability}

We showed earlier that the variances within each generation are a measure of genotype interaction with the microenvironmental conditions on the particular scale being used, but that this interaction may be eliminated where there is a simple scalar relationship with the means. The angular transformation reduces any such relationship and, by the formula $821 /(n+$ $0.5)$, gives theoretical error variances of 40.04 for the main Cavalli cross and 78.19 for the other experiments (d.f. $=\infty$ ), which may be compared with the observed variances of the non-segregating generations in the Cavalli crosses (table 5).

Interaction with the microenvironment occurs even in the strictly controlled main Cavalli cross, where there is a general potence for low variability. Although there are several similarities with the analyses of the means in table $3 a$, such as the reciprocal differences in the activity and preening variances of the males, the fresh Cavalli cross proves that such effects are not merely the results of a correlation with the means. Here a far wider range of environmental variables could increase the interindividual differences, but the rise in variance is largely confined to the inbred lines, resulting in potence for low variability of preening, as well as of activity, despite the absence of dominance for preening itself. The genotypes may be reacting differently to environmental factors either within each culture vial, or between the replicate cultures. In the diallel cross, where the additional transformation had ensured that there was no correlation with the means, N.C.II analyses of the within vial variances revealed a paternal effect on both measures (male parent MS, $\mathrm{P}<5$ per cent. for preening and $\mathrm{P}<1$ per cent. for activity). The differences between the variances of replicate vials provide error terms for this analysis of $E_{1}$, the within culture effects, but there is no suitable error when one studies the variances between all the individuals within each of the 36 crosses, disregarding the fact that they come from different vials, in order to determine the contribution of $E_{2}$, the differences between replicate cultures, to the overall variability. Here, the 
only possibility is to test the $a$ and $b$ mean squares of the Hayman analysis against the $c$ and $d$ items respectively, the tests Wearden (1964) recommended in the presence of maternal effects. On both measures, the females showed potence for low variability (the $b_{1} \mathrm{MS}, \mathbf{P}<1$ per cent), with more than 20 of the $30 \mathrm{~F}_{1} \mathrm{~s}$ being less variable than either parent. Among the males, on the other hand, there were additive effects and strain differences in dominance (the $a$ and $b_{3}$ items both $\mathrm{P}<1$ per cent.) plus, in the case of activity, some reciprocal differences in the variances, since the $c$ MS was significantly larger ( $\mathrm{P}<5$ per cent.) than the $d$ MS.

TABLE 5

The variances of the activity and preening of Edinburgh $(E), 6 C I L(C)$ and their reciprocal $F_{1} s$ in the Cavalli crosses. These statistics are based on the angular-transformed data and have standard errors calculated as $\sqrt{2 \text { var. }{ }^{2} /(d . f .-1)}$, where d.f. $=159,35$ and 53 for the main, fresh vial and original bottle condition experiments respectively

Experiment

\begin{tabular}{|c|c|c|c|c|c|}
\hline \multirow[b]{2}{*}{ Sex } & \multirow[b]{2}{*}{ Measure } & & \\
\hline & & Generation & Main & Fresh vial & Original bottles \\
\hline \multirow{8}{*}{ Male } & \multirow{4}{*}{ Activity } & $\mathbf{E}$ & $188.5 \pm 21 \cdot 1$ & $335 \cdot 3 \pm 81 \cdot 2$ & $307 \cdot 1 \pm 60 \cdot 2$ \\
\hline & & $\mathrm{C}$ & $188 \cdot 3 \pm 21 \cdot 2$ & $307 \cdot 7 \pm 74.5$ & $416 \cdot 0 \pm 81 \cdot 6$ \\
\hline & & $\mathrm{E} \times \mathrm{C}$ & $140 \cdot 6 \pm 15 \cdot 8$ & $106 \cdot 7 \pm 25 \cdot 7$ & $301 \cdot 6 \pm 59 \cdot 0$ \\
\hline & & $\mathrm{C} \times \mathrm{E}$ & $117 \cdot 7 \pm 13 \cdot 1$ & $234 \cdot 9 \pm 56 \cdot 7$ & $280 \cdot 9 \pm 54 \cdot 9$ \\
\hline & \multirow{4}{*}{ Preening } & & & & $227.1+44.5$ \\
\hline & & $\frac{\mathrm{E}}{\mathrm{C}}$ & $128 \cdot 3+14 \cdot 4$ & $\begin{array}{l}204 \cdot 1 \pm 49.7 \\
177 \cdot 2+42.9\end{array}$ & $\begin{array}{l}22 \cdot \cdot 1 \pm 44 \cdot 3 \\
226 \cdot 3+44 \cdot 3\end{array}$ \\
\hline & & $E \times C$ & $161 \cdot 5+18 \cdot 2$ & $86 \cdot 1+20 \cdot 9$ & $163 \cdot 8 \pm 31 \cdot 9$ \\
\hline & & $\mathrm{C} \times \mathrm{E}$ & $110 \cdot 5 \pm 12 \cdot 4$ & $114 \cdot 4 \pm 27 \cdot 0$ & $200 \cdot 2 \pm 39 \cdot 2$ \\
\hline \multirow{8}{*}{ Female } & \multirow{4}{*}{ Activity } & $\mathbf{E}$ & $186 \cdot 9+21 \cdot 0$ & $382 \cdot 0+92 \cdot 4$ & $237 \cdot 6+46 \cdot 5$ \\
\hline & & C & $136 \cdot 0 \pm 15 \cdot 5$ & $499 \cdot 9 \pm 121 \cdot 0$ & $308 \cdot 6 \pm 60 \cdot 4$ \\
\hline & & $\mathrm{E} \times \mathrm{G}$ & $110 \cdot 8 \pm 12 \cdot 4$ & $242 \cdot 2 \pm 58 \cdot 7$ & $392 \cdot 0 \pm 76 \cdot 7$ \\
\hline & & $\mathrm{C} \times \mathrm{E}$ & $121 \cdot 2 \pm 13 \cdot 7$ & $400 \cdot 3 \pm 97 \cdot 1$ & $445 \cdot 6 \pm 87 \cdot 3$ \\
\hline & \multirow{4}{*}{ Preening } & & & & \\
\hline & & C & $90 \cdot 2+10 \cdot 1$ & $\begin{array}{l}200 \cdot 6 \pm 48 \cdot 6 \\
224 \cdot 5+54 \cdot 3\end{array}$ & $\begin{array}{l}142 \cdot 7 \pm 27 \cdot 8 \\
202 \cdot 0+39 \cdot 4\end{array}$ \\
\hline & & $E \times C$ & $107 \cdot 8+12 \cdot 1$ & $125 \cdot 8+30 \cdot 3$ & $239.2+46.9$ \\
\hline & & $\mathrm{C} \times \mathrm{E}$ & $99 \cdot 7 \pm 11 \cdot 2$ & $123 \cdot 5 \pm 29 \cdot 8$ & $253 \cdot 1 \pm 49 \cdot 6$ \\
\hline
\end{tabular}

Flies left in the original bottles are exposed to many random environmental factors, such as food availability and infection by microflora (Connolly, 1966), so that even larger variances are to be expected. But table 5 shows that only the $\mathrm{F}_{1}$ s have increased in variability to such an extent that, among the females, there is potence for high variability for activity and, to a lesser extent, for preening. It will be recalled that the activity of the $6 \mathrm{C} / \mathrm{L}$ females rose over the three days of testing and similarly their variance decreased over this period, from 383.5 to 195.8 and to 153.2 for activity (homogeneity $\chi_{2}^{2}=4.01, P<10$ per cent.) and from 294.5 to 125.5 and to 93.0 for preening $\left(\chi_{2}^{2}=6.38, P<5\right.$ per cent.). Such changes were absent in the other genotypes.

After the additional transformation the activity variances of the diallel cross were still heterogeneous (table 1 ). Because $\chi^{2}$ s are additive, subtraction of the non-significant $\chi_{63}^{2}$ of the $4 \times 4$ diallel from the corresponding $\chi_{143}^{2}$ of the $6 \times 6$ leaves a $\chi_{80}^{2}$ of $114.01 \quad(P<1$ per cent.) for the contribution to the heterogeneity of variance of crosses involving Oregon and Samarkand. The average variance within the $6 \times 6$ diallel cross was less than the $4 \times 4-3712 \cdot 3 \pm$ 
268.6 compared with $4388 \cdot 2 \pm 211 \cdot 4$ on the transformed scale-and both the activity and preening variances were significantly smaller $(\mathrm{P}<1$ per cent. on the second than on the first day of testing, while the N.C.II analysis showed the father's genotype become more important, especially among the male progeny (the day $\times$ male parent $\mathrm{MS}$ and its sex interaction were both significant at $\mathrm{P}<1$ per cent.).

\section{The mortality rates of $6 C / L$ and Edinburgh}

An animal's behaviour is fundamental to its survival (Roberts, 1967, p. 343) and, before discussing our results on activity and preening, we may usefully consider what is known of the viability of $6 \mathrm{C} / \mathrm{L}$ and Edinburgh. Some differences in mortality were observed during the present experiments; the poor viability among the males in the fresh Cavalli cross, which prevented their being tested on a third day, was confined largely to $6 \mathrm{C} / \mathrm{L}$ and this strain suffered more than Edinburgh through being left in the original bottles. Here all the cultures, but especially those of $6 \mathrm{C} / \mathrm{L}$, were becoming more choked every day with the sticky secretions of the flies with the result that many adhered to the walls. However, it would have been impossible to make systematic counts of the numbers dying in this way without causing considerable disturbance to the survivors.

Three separate experiments have therefore been performed to study viability. Firstly, 10 samples of 50 larvae from each strain were incubated at $25^{\circ}$ C. and the 5 per cent. fiducial limits for the percentage emerging were 13-22 per cent. for Edinburgh and 76-85 per cent. for $6 \mathrm{C} / \mathrm{L}$. Those Edinburgh flies which did emerge were less viable because, in a second experiment where samples of 30 newly emerged females were left in culture vials, only 49 per cent. of Edinburgh were still alive after 7 days, compared with 79 per cent. for $6 \mathrm{C} / \mathrm{L}$. This difference was significant at $\mathrm{P}<0.1$ per cent. and seems to contradict our observations on the culture bottles, until the third, more extensive, experiment is considered.

Within 12 hours of emergence, males and females from $6 \mathrm{C} / \mathrm{L}$ and Edinburgh were put into groups of 10 of the same sex in culture vials with fresh food (the same density as in the main Cavalli cross). The numbers surviving after 72 and 144 hours were noted, six of the 12 groups in each category being transferred to vials with fresh food at 72 hours, while the others were left in the same vials.

Table 6 gives the percentage surviving in the three conditions used:

TABLE 6

The percentage of Edinburgh $(E)$ and $6 C / L(C)$ surviving until (a) 72 hours after eclosion, and until 144 hours, (b) with and (c) without transfer to fresh culture vials.

(a) 72 hours

\begin{tabular}{lcc}
\multicolumn{1}{c}{ Sex } & E & G \\
Male & 86 & 95 \\
Female & 70 & 91 \\
Heterogeneity & $\chi_{3}^{2}$ & 4.04 n.s.
\end{tabular}

(b) 144 hours, fresh vials

$\begin{array}{cc}\mathrm{E} & \mathrm{C} \\ 90 & 89 \\ 82 & 86\end{array}$

0.44 n.s. (c) 144 hours, original vials

$\begin{array}{cc}\mathrm{E} & \mathrm{C} \\ 70 & 42 \\ 85 & 75\end{array}$

$14 \cdot 97 * *$

The significant heterogeneity between the four results in $(c)$ can be partitioned into the difference between the two strains $\left(\chi_{1}^{2}=8 \cdot 47^{* *}\right)$ and between the two sexes $\left(\chi_{1}^{2}=5 \cdot 31^{*}\right)$, leaving no significant interaction.

** $\mathrm{P}<1$ per cent.; *P = 1-5 per cent.; n.s. = not significant. 
(a) the percentage alive at 72 hours of the 120 flies of each sex and strain put in the vials at emergence;

(b) the percentage alive at 144 hours of those transferred to fresh vials at 72 hours; and

(c) the corresponding percentage surviving until 144 hours, of those alive in the original vials at 72 hours and not subsequently transferred.

Expressing the final results as percentages of those surviving at an intermediate stage takes into account the higher initial mortality of Edinburgh. At 72 hours $6 \mathrm{C} / \mathrm{L}$ was slightly superior to Edinburgh and the relative mortalities were unaltered over the next 72 hours, provided the flies were transferred to fresh vials. Otherwise, when left in the less favourable conditions of the original vials, the death rate of $6 \mathrm{C} / \mathrm{L}$ was far higher than that of Edinburgh and the males of the two strains proved the less hardy sex, confirming both our observations on the culture bottles and the evidence of Parsons (1966) on sex differences in the longevity of $D$. melanogaster.

\section{Discussion}

The genetical control of activity and preening is certainly not simple in that it varies considerably between different experimental conditions. The control is sensitive to many types of environmental influence and particular emphasis has been put on two forms of environmental effect, the genotypic interaction with the microenvironment and the role of the maternal genotype, because these factors can confound a simple genetical analysis. At least the analytical procedures of biometrical genetics are capable of detecting and estimating such influences, in contrast to the selection studies of activity, where, in addition, the increased sensitivity to the environment with increasing homozygosity can lead to considerable fluctuation in performance between successive generations (Connolly, 1966).

\section{Natural selection and the genetical architecture}

The main advantage of biometrical genetical techniques lies in providing comprehensive information on the genetical architecture, allowing extrapolation to the action of natural selection on behavioural traits. There is little additive variation for activity, but rather directional dominance for high activity, reinforced by duplicate interaction in the males where many genotypes would otherwise be far less active. This form of genetical architecture is characteristic of traits for which there has been directional selection, rather than stabilising selection for an intermediate optimum (Kearsey and Kojima, 1967). However, the effects on viability of natural selection may become apparent only in inferior conditions, for example during the testing of flies from the original bottles, where the selective advantage of high activity is shown by the rise in activity among the survivors, attributable to the less active flies being more likely to adhere to the walls of their container. Such selection against low activity is also consistent with the highest mortality in the diallel cross being found in Samarkand, the least active strain in the fresh conditions, and the $6 \mathrm{C} / \mathrm{L}$ strain of the Cavalli cross showing the most marked rise in activity, since we have seen that this strain is far less viable than Edinburgh when reared in the original culture bottles.

However, the low activity and low viability of Samarkand, and initially too of Edinburgh adults, are likely consequences of their poor larval-adult 
survival rates of 8-15 per cent. and 13-22 per cent. respectively. Thus the level of activity may itself depend upon viability and we cannot conclude that high activity necessarily increases the probability of survival. Inbreeding may also influence the relationship between viability and the genetical architecture of activity, although there is not the simple decrease in activity with increased inbreeding which Harland and Jackson (1958) proposed; for instance, $6 \mathrm{C} / \mathrm{L}$ had been inbred for 150 generations at the time of the diallels and Samarkand for 280, but Wellington, which was far more active than the latter, for 750 generations. It has frequently been demonstrated in Drosophila (Jinks and Mather, 1955; Breese and Mather, 1960; Parsons, 1966) that heterozygotes have a higher viability than inbred lines and that their performance on both morphological and behavioural measures is far less affected by environmental changes. Therefore, the changes in the genetical control, and especially the reversal of the direction of dominance, between the fresh and original conditions of rearing, are due to the high mortality of the less active flies affecting the inbred lines but not the $\mathrm{F}_{1} \mathrm{~s}$.

Preening depends more on the maternal genotype than on autosomal genetical control and we can demonstrate that these complex maternal effects are the outcome of factors influencing culture density. Because preening is used to fend off other flies, perhaps to space them over the available food or egg-laying space (Connolly, 1968; Hay, 1972b), the level of preening should be related to the density of flies in the cultures. Density in turn is influenced by several factors known to be controlled by the maternal genotype, such as egg hatchability and also egg-laying capacity, for which $F_{1}$ mothers show heterosis (Barnes, 1968). Hence, additive maternal effects on egg hatchability should be a greater influence on preening in the fresh conditions than in the low-density main Cavalli cross, except for any effects acting at the larval stage. Competition does occur during the pre-adult stages (Kearsey and Barnes, 1970) and a significant ( $\mathbf{P}=1$ per cent.) change in preening was found between the preening of the early- and late-emerging male flies in the main Cavalli cross, although the cause is unknown and there were no interactions with the genotypes. Compared with the flies in fresh vials, the presence of an $F_{1}$ maternal effect $[\mathrm{hm}]$, when the flies are left in the original bottles, results from the procedural differences between the conditions. By not transferring the flies to fresh vials on the second day after the cultures began to emerge, any extra eggs laid by the $F_{1}$ mothers in the bottles will have a chance to emerge and hence to influence the culture density in the period before testing. Certainly, preening is unlikely to be only the "cleaning " movements of Szebenyi (1969), because it has not increased appreciably in these conditions, despite the greater likelihood of debris adhering to the flies.

In the introduction we mentioned the difficulty in psychogenetics of determining just what is being measured and the distinction of Connolly (1967) between spontaneous activity and reactivity becomes important when the experimental procedure is changed. The first response of the flies to the stimulus is usually activity, so that preening will be less where this reaction is greater. Thus, when we score activity over a longer period (the main Cavalli cross) or where the stimulus is less intense (the dishes of the comparison Cavalli cross) or where the physical conditions of the flies is adversely affected by the environment (the original culture bottles), preening 
is increased and we are measuring spontaneous activity rather than reactivity. The dominance for low preening in these experiments need not imply this behaviour is adaptive in the same way as high activity, but only that the measures are negatively correlated, so that the most active flies are less likely to preen. That is, there is dominance for high reactivity, not detectable in those conditions where the initial stimulation has a greater effect on all flies.

The reverse interpretation, that low preening is advantageous for survival and that the dominance for high activity results from the negative correlation, is less likely because preening neither shows a genetical architecture consistent with directional selection nor are there changes between the different conditions which can be related to viability differences, in the way possible for activity. Also, the experiment on cage populations, mentioned in the introduction, provides independent evidence for the adaptive value of high activity and there, the males of the cage samples, unlike the inbred lines, did not preen more than their females when grouped together, since such reactions to other males would interfere with the normal courtship behaviour directed to females (Connolly, 1968). Similarly, in the main Cavalli cross of the present experiment, the males were far less active than the females and did not show the same mirror-image relationship between the models, suggesting they are less reactive to the physical stimulation administered prior to testing. Reactivity of this type is known to reduce the mating success of males (Manning, 1961), and the slow mating speed of $6 \mathrm{C} / \mathrm{L}$ males (Fulker, 1966) has previously been related to their high reactivity (Hay, 1972a). Furthermore, Kaplan and Trout (1969) used the number of "fly specks" deposited in the vials as an index of reactivity, and high reactivity would therefore explain why the culture bottles of the very active $6 \mathrm{C} / \mathrm{L}$ flies became fouled more rapidly, leading to a higher mortality than among the Edinburgh flies, unless transferred to fresh vials.

The genetical architecture has enabled us to make inferences about our measures of activity and preening but, in view of the many points of correspondence between activity and preening in the analyses of the variances, we may regard sensitivity to the environment as a third measure that can be extracted from these data. Such a factor is not unrealistic because, for example, Perkins and Jinks (1968) demonstrated that the means and variances of flowering time in Nicotiana rustica are controlled by two independent genetical systems and Dobzhansky and Levene (1955) showed in $D$. pseudoobscura that sensitivity to both macro- and micro-environmental fluctuations was negatively correlated with viability. Similarly, because of its poor larval-adult survival rate and lower initial adult viability, Edinburgh shows a greater variability between individuals on both measures in the main Cavalli cross than does the $6 \mathrm{C} / \mathrm{L}$ strain. This pattern is, however, reversed in the original Cavalli cross, the condition where the $6 \mathrm{C} / \mathrm{L}$ flies have a higher mortality rate. Viability is therefore negatively correlated with variability on both our measures and hence the potence for low variability in the fresh conditions may result from the superior viability of the heterozygotes, mentioned previously. On the other hand, in the original cultures there is potence for high variability, the average variance of the crosses to Oregon and Samarkand is less, and 6C/L shows a decrease in variance over the three days of testing. These effects may all result from 
the poor viability of these inbred lines, in the same way that Kearsey and Barnes (1970) attributed the low variability of sternopleural chaetae number in $D$. melanogaster, when reared under intense competition, to selection against the extreme phenotypes.

Male Drosophila are less hardy than the females and not only do they show more extensive genetical control of the variances but also their behaviour is consistently more dependent upon the maternal genotype. In the case of females, most of the differences between reciprocals in the diallel cross in the original bottles involved Oregon or Samarkand as one parent, the only exception being the "paternal" interaction for which $6 \mathrm{C} / \mathrm{L}$ was responsible. Hence we may conclude viability is negatively correlated with sensitivity to maternal, as well as to microenvironmental, influences and indeed several maternal effects were found in the analyses of the variances.

Clearly, there are many complications in the genetical analyses of Drosophila behaviour. When the flies are reared in poor conditions, phenotypic selection can extensively alter the outcome of genetical analyses and even the nature of the trait under consideration may change when the environment or testing procedure is altered. Repeating the same experiment under different conditions would therefore seem essential, before any generalisations can be made about the adaptive importance of the trait under consideration.

\section{Implications for biometrical genetical analysis}

Having seen how biometrical genetics can assist in the study of behaviour, we can consider what implications these findings may have for the methods of genetical analysis.

Firstly, is it necessary to rescale data? The analyses of the variances have many similarities with those of the activity means, but the negative correlation between activity and variability (table 1) is due to selection favouring high activity and low variability, and not to a simple effect of the scale of measurement, where for instance very high means may impose an upper limit on the range of the variances. Even after rescaling the diallel cross data, it was still possible to detect the genetical differences in variability and the results of the Hayman and N.C.II analyses of the means were unaffected. This illustrates the stability of the analysis of variance to failure of the assumptions underlying parametric tests and the estimation of parameters from the first-degree statistics of the Cavalli crosses shares this advantage. With dominance for high activity there should be a negative correlation between the means and variances for the 14 generations, since the backcross to the more active parent will have a larger mean but a smaller withinfamily variance. Transformations of 1.8919 and 0.3300 were derived for the activity and preening data respectively of the fresh Cavalli cross, in the way detailed previously for the diallel cross data (table 1), and proved effective in eliminating significant correlations between the means and variances, as well as any heterogeneity of variance. But the $A, B$ and $C$ scaling tests, perfect fit $m,[d],[h],[i],[j],[l]$ models calculated from the generation means after averaging over reciprocals, and even the best fit models of table 3, gave exactly the same significant results both before and after the additional transformation. Although the transformations affected the relative magnitude of the parameters, heterosis was still found for activity, as well as potence for low variability on both measures and even the reciprocal 
differences in the $F_{1}$ variances. Similarly, Newell (1970) found square root and natural $\log$ transformations had no effect on the detection of $[d]$ and $[h]$ and Copp and Wright (1952) reported that parent/offspring correlations were unaffected by a transformation, derived systematically from the data to meet the genetical criteria for scaling, namely homogeneity of variance among the non-segregating generations and absence of epistasis in the $\mathrm{A}, \mathrm{B}$ and $\mathrm{C}$ scaling tests.

Our transformation is determined directly from the data and is capable of removing the correlation between means and variances in many types of data. In this way it fulfils the first of the two genetical criteria for scaling cited above, but the detection of epistasis by the $\mathrm{A}, \mathrm{B}$ and $\mathrm{C}$ scaling tests is unaffected. However, these two criteria may sometimes be incompatible (Mather and Jinks, 1971) and we showed that genotype-microenvironmental interaction, leading to heterogeneity of the variances, can also influence the S.E.s of the A, B and C scaling tests; in fact, it was possible to detect epistasis for activity in the main Cavalli cross even though none of the A, $\mathrm{B}$ or $\mathrm{C}$ tests differed from zero. Furthermore, potence for low variability was demonstrated in the fresh diallel cross, despite the absence of any overall heterogeneity among the 36 variances after the additional transformation.

It would appear, therefore, that the usual criteria for scaling are not sufficiently rigorous as to exclude genotype-environmental interaction and epistasis completely, so that an additional transformation may be of little help in the analysis, except possibly to eliminate from the study of the variances the complication of correlation with the means. But rescaling proved unnecessary in these data only because no attempt was made to calculate parameters from the second-degree statistics, which in turn has meant that two items of information were unobtainable. Firstly, we could not allow for the effects of gene association or dispersion when heterosis was detected in the Cavalli crosses, and secondly, we could not partition the components of variation. This second point is crucial in model fitting, because of the paradox in weighted least squares analysis that, the larger the experiment, the more difficult it is to obtain an adequate model, since the standard errors of the generation means are smaller and the models must therefore be more precise. This can mean that, with successive modelfitting to minimise the $\chi^{2}$, many parameters may be added which are trivial in that they account for only a small proportion of the variation, although their estimates appear large as a result of inflation by negative correlations; for example, the relationship of $[\hat{h}]$ and $[\hat{e}]$ in the female data of the main Cavalli cross (table $3 a$ ). A rough indication of the importance of a parameter is provided by the amount by which its inclusion in the model reduces the $\chi^{2}$ although, in practice, it frequently happens that once the model contains more than three or four parameters, any additional parameter may be highly significant, compared with its S.E., but has little effect on the $\chi^{2}$. If estimates of $\mathrm{D}, \mathrm{H}$ and $\mathrm{E}$ are available, one can then calculate how much of the total variation remains to be accounted for by epistasis, maternal effects, etc. (see, for example, Perkins and Jinks, 1970) and avoid adding unnecessary parameters. The only way to reduce the role of correlations between parameters in the estimation from first-degree statistics is to rear additional generations and hence sample a wider range of coefficients for each parameter (compare, for instance, the coefficient 
for $[i],[j]$ and $[l]$ in our table 2 with those given by Jinks and Perkins (1969 table 3) for digenic interactions in 21 different generations).

On the other hand, we have been able, without fitting second-degree models, to study genotype-microenvironmental interaction and maternal effects in considerable detail, for which both the Gavalli and diallel crossing schemes are required. With the Cavalli cross models, complex maternal effects can be investigated far more easily than by the elaborate analyses required to obtain such information from the diallel cross (Durrant, 1965).

However, Allard (1956) has shown that the cross-classified Hayman analysis of the diallel provides tests for the interaction between the genetical components of variation and environmental factors (for example, the successive days of testing in our data), which are not so practicable in the Cavalli cross. Also, a comprehensive analysis of the inter-individual variances is possible in the diallel (Broadhurst and Jinks, 1966), and there are sufficient crosses to allow conclusive tests for the presence of any correlation between the means and variances which could affect the results of such an analysis. The four non-segregating generations of the Cavalli cross provide less information on the variability and the analysis becomes far more difficult if the other generations are studied (Perkins and Jinks, 1970). Both the Hayman and N.C.II analyses are clearly essential to the study of diallel data; the former is needed to study the genetical variation and the latter to determine, by the relative magnitudes of the maternal and paternal mean squares, whether maternal effects are acting in the same or opposite direction to the genetical influences. When there are maternal effects, the N.G.II cannot detect genetical variation and the model given by Wearden (1964) for maternal effects in this analysis does not hold for the complex maternal interactions present in our data, which led, for instance, to the failure to detect dominance in the male preening data of the fresh diallel cross.

Much can therefore be learned from these analyses of the diallel, without calculating the components of genetical variation. Nevertheless, it must not be assumed that the analysis of second-degree statistics is unnecessary, since the approaches based on first- and second-degree statistics are complementary, rather than alternative (Mather and Jinks, 1971, p. 126). Firstdegree statistics are adequate for the study of maternal effects, but to provide convincing evidence for epistasis and sex-linkage without using the variances, further experiments would be required. Unlike the Cavalli cross analysis, a Triple Test cross based on Edinburgh and $6 \mathrm{C} / \mathrm{L}$ would provide a test for epistasis which is independent of additive and dominance effects. Chromosome assay has been employed in psychogenetics and could detect interchromosomal interactions (c.f. Kearsey and Kojima, 1967), and also separate the influences of the sex chromosome from those of the maternal genotype, a distinction which the greater maternal effects in the male progeny may have made equivocal in these data. Such an assay of the substitution lines between $6 \mathrm{C} / \mathrm{L}$ and Edinburgh is currently in progress and further population cage studies are planned, to confirm the inferences from the genetical architecture about the action of natural selection on the activity and preening of Drosophila.

\section{Summary}

1. The genetical control of the activity and preening behaviour of $D$. melanogaster was studied by diallel crosses between six inbred lines and by $\times 2$ 
estimation of first-degree parameters from the means of the parental, $\mathbf{F}_{1}$, $\mathbf{F}_{\mathbf{2}}$ and first back-cross generations, derived by crossing the two most extreme strains.

2. When reared in a favourable environment, both sexes showed directional dominance for high activity, with duplicate interaction and a dispersed gene distribution ensuring uniformly high activity in most generations.

3. In crowded conditions, the level of preening in females could be related to additive maternal effects on egg hatchability and to dominance maternal effects on fecundity; in males preening was influenced by an interaction between the maternal and progeny genotypes during the preadult stages.

4. Leaving the flies in the original culture bottles for the 7-day period between eclosion and testing led to a high mortality among the males of certain strains and altered the genotypic differences in the behaviour of both sexes.

5. The greatest mortality occurred among the less active flies of the inbred lines, resulting in dominance for low activity and suggesting the selective benefits of the dominance for high activity found previously.

6 . Interaction between the genotypes and the microenvironment made estimation of second-degree parameters impossible. Potence for low variability was generally found, except among the flies left in the original culture bottles where the extreme phenotypes among the inbred lines had a poorer viability, reversing the direction of potence.

7. Sensitivity to both maternal and microenvironmental effects was negatively correlated with viability.

8. The question of the rescaling of genetical data is discussed in respect of a transformation, derived from the data and capable of removing all the correlation between means and variances.

9. The advantages of the Hayman and N.C.II analyses of variance of diallel data are compared with those of genetical analysis by fitting models to the generation means; both types of analysis are required in order to detect maternal and genotype-microenvironmental influences and both are unaffected by failure of the genetical criteria for the rescaling of data. However, these analyses of the means cannot eliminate the need to study seconddegree statistics.

Acknowledgments.-The work was performed during the tenure of a Science Research Council postgraduate studentship and forms part of a programme of research in psychogenetics in the Departments of Genetics and Psychology, supported by a grant from the Medical Research Council. The author acknowledges the guidance of Dr M. J. Kearsey and the helpful comments on the manuscript given by Professors P. L. Broadhurst and J. L. Jinks.

\section{REFERENCES}

ALLARD, R. W. 1956. The analysis of genetic-environmental interactions by means of diallel crosses. Genetics, 41, 305-318.

BARNes, B. w. 1968. Maternal control of heterosis for yield in D. melanogaster. Heredity, 23, $563-572$.

BREESE, E. L., AND MATHER, K. 1960. The organisation of polygenic activity within a chromosome in Drosophila. II. Viability. Heredity, 14, 375-399.

BROADHURST, P. L., AND JINKS, J. L. 1961. Biometrical genetics and behaviour: re-analysis of published data. Psychol. Bull., 58, 337-362.

BROADHURST, P. L., AND JINKS, J. L. 1966. Stability and change in the inheritance of behaviour in rats: a further analysis of statistics from a diallel cross. Proc. roy. Soc. B., 165, 450-472. 
CAVALII, L. L. 1952. An analysis of linkage in quantitative inheritance. In Quantitative Inheritance, Ed. E. C. Reeve and C. H. Waddington, 135-144. London: H.M.S.O.

connoliy, к. J. 1966. Locomotor activity in Drosophila. II. Selection for active and inactive strains. Anim. Behav., 14, 444-449.

conNoliy, K. J. 1967. Locomotor activity in Drosophila. III. A distinction between activity and reactivity. Anim. Behav., 15, 149-152.

connolly, к. J. 1968. The social facilitation of preening behaviour in D. melanogaster. Anim. Behav., 16, 385-391.

COPP, L. G. L., AND WRIGHT, G. M. 1952. The inheritance of kernel weight in a Triticum Vulgare cross. Heredity, 6, 187-199.

DOBZHANSKY, TH., AND LEVENE, H. 1955. The genetics of natural populations. 24. Developmental homeostasis in natural populations of $D$. pseudoobscura. Genetics, 40, 797-808.

DURRANT, A. 1965. Analysis of reciprocal differences in diallel crosses. Heredity, 20, 573-607.

EAVES, L. J. 1969. The genetic analysis of continuous variation: a comparison of experimental designs applicable to human data. Br. 7. Math. and Stat. Psychol., 22, 131-147.

eWtng, A. w. 1963. Attempts to select for spontaneous activity in D. melanogaster. Anim. Behav., 11, 369-378.

EWING, A. W. 1967. Genetics and activity in D. melanogaster. Experientia, 23, 330-335.

FULKER, D. w. 1966. Mating speed in male D. melanogaster: a psychogenetic analysis. Science, 153, 203-205.

PULKER, D. w. 1970. Maternal buffering of rodent genotypic responses to stress: a complex genotype-environment interaction. Behav. Genetics, 1, 119-124.

harLAND, s. C., AND JACKSON, A. R. H. 1958. Advantage of the white eye mutant of $D$. melanogaster over the wild type in an artificial environment. Heredity, 12, 27-36.

HAY, D. A. 1972a. Behavioural rhythms in cultures of immature D. melanogaster. Experientia, in press.

HAX, D. A. $1972 b$. Recognition by $D$. melanogaster of individuals from other strains or cultures: support for the role of olfactory cues in selective mating? Evolution, in press.

HAYMAn, в. 1. 1954. The analysis of variance of diallel crosses. Biometrics, 10, 235-244.

HILL, J. 1964. Effects of correlated gene distribution in the analysis of diallel crosses. Heredity, $19,27-46$.

JINKs, J. L. 1954. The analysis of continuous variation in a diallel cross of Nicotiana rustica varieties. Genetics, 39, 767-788.

JINks, J. L., AND JONES, R. M. 1958. Estimation of the components of heterosis. Genetics, 43, 223-234.

JINKS, J. L., AND MATHER, K. 1955. Stability in development of heterozygotes and homozygotes. Proc. roy. Soc. B., 143, 561-578.

JINKS, J. L., AND PERKINS, J. M. 1969. The detection of linked epistatic genes for a metrical trait. Heredity, 24, 465-474.

KAPLAN, w. D., AND TROUT, w. E. 1969. The behavior of four neurological mutants of Drosophila. Genetics, 61, 399-409.

KEARSEY, M. J., AND BARNES, B. w. 1970. Variation for metrical characters in Drosophila populations. II. Natural selection. Heredity, 25, 11-21.

KEARSEY, M. J., AND KOJIMA, K.-I. 1967. The genetic architecture of body weight and egg hatchability in $D$. melanogaster. Genetics, 56, 23-37.

ManNing, A. 1961. The effects of artificial selection for mating speed in D. melanogaster Anim. Behav., 9, 82-92.

MATHER, K. 1966. Variability and selection. Proc. roy. Soc. B., 164, 328-340.

MATHER, K. 1967. Complementary and duplicate gene interactions in biometrical genetics. Heredity, 22, 97-103.

MATHER, K., AND JiNKS, J. L. 1971. Biometrical Genetics (2nd edn.). London: Chapman \& Hall.

MOSTELIER, F., AND Youtz, c. 1961. Tables of the Freeman-Tukey transformations for the binomial and Poisson distributions. Biometrika, 48, 433-440.

MURPHEY, R. M., AND HALL, C. F. 1969. Some correlates of negative geotaxis in D. melanogaster. Anim. Behav., 17, 181-185.

NEWELL, T. G. 1970. Three biometrical genetic analyses of activity in the mouse. F. comp. physiol. Psychol., 70, 37-47.

PARSONS, P. A. 1966. The genotypic control of longevity in D. melanogaster under two environmental regimes. Aust. F. biol. Sci., 19, 587-591.

PERKINS, J. M. 1970. Environmental and genotype-environmental components of variability. VI. Diallel sets of crosses. Heredity, 25, 29-40. 
PERKINS, J. M., AND JINKS, J. L. 1968. Environmental and genotype-environmental components of variability. III. Multiple lines and crosses. Heredity, 23, 339-356.

PERKINS, J. M., AND JINKS, J. L. 1970. Detection and estimation of genotype-environmental, linkage and epistatic components of variation for a metrical trait. Heredity, 25, 157-177. ROBERTS, R. G. 1967. Implications of behavior genetics for genetics. In Behavior-Genetic Analysis, Ed. J. Hirsch, 340-343. New York: McGraw-Hill.

SIEGEL, J. M. 1967. Heritability and threshold determinations of the optomotor response in D. melanogaster. Anim. Behav., 15, 299-306.

SZEBenyi, A. L. 1969. Cleaning behaviour in D. melanogaster. Anim. Behav., 17, 641-651. WEARDEN, s. 1964. Alternative analysis of the diallel cross. Heredity, 19, 669-680. 